\title{
Intraovarian vascular enhancement via stromal injection of platelet-derived growth factors: Exploring subsequent oocyte chromosomal status and in vitro fertilization outcomes
}

\author{
Samuel H. Wood', E. Scott Sills ${ }^{1,2}$ \\ 'Gen 5 Fertility Center, San Diego, CA, USA; ${ }^{2}$ Applied Biotechnology Research Group, University of Westminster, London, UK
}

The inverse correlation between maternal age and pregnancy rate represents a major challenge for reproductive endocrinology. The high embryo ploidy error rate in failed in vitro fertilization (IVF) cycles reflects genetic misfires accumulated by older oocytes over time. Despite the application of different follicular recruitment protocols during IVF, gonadotropin modifications are generally futile in addressing such damage. Even when additional oocytes are retrieved, quality is frequently poor. Older oocytes with serious cytoplasmic and/or chromosomal errors are often harvested from poorly perfused follicles, and ovarian vascularity and follicular oxygenation impact embryonic chromosomal competency. Because stimulation regimens exert their effects briefly and immediately before ovulation, gonadotropins alone are an ineffective antidote to long-term hypoxic pathology. In contrast, the tissue repair properties (and particularly the angiogenic effects) of platelet-rich plasma (PRP) are well known, with applications in other clinical contexts. Injection of conventional PRP and/or its components (e.g., isolated platelet-derived growth factors as a cell-free substrate) into ovarian tissue prior to IVF has been reported to improve reproductive outcomes. Any derivative neovascularity may modulate oocyte competence by increasing cellular oxygenation and/or lowering concentrations of intraovarian reactive oxygen species. We propose a mechanism to support intrastromal angiogenesis, improved follicular perfusion, and, crucially, embryo ploidy rescue. This last effect may be explained by mRNA upregulation coordinated by PRP-associated molecular signaling, as in other tissue systems. Additionally, we outline an intraovarian injection technique for platelet-derived growth factors and present this method to help minimize reliance on donor oocytes and conventional hormone replacement therapy.

Keywords: Fertilization in vitro; Hypoxia; Platelet rich plasma

\section{Introduction}

Female age is the single most important variable in the determination of pregnancy outcomes, for advanced reproductive technolo-

Received: November 1, 2019· Revised: December 17, 2019.

Accepted: December 24, 2019

Corresponding author: E. Scott Sills

Gen 5 Fertility Center, Office for Reproductive Research, 3420 Carmel Mountain Rd, Suite 200, San Diego, CA 92121, USA

Tel:+1-949-899-5686 Fax:+1-858-225-3535 E-mail:drsills@CAGivf.com

This is an Open Access article distributed under the terms of the Creative Commons Attribution Non-Commercial License (http://creativecommons.org/licenses/by-nc/4.0/) which permits unrestricted non-commercial use, distribution, and reproduction in any medium, provided the original work is properly cited. gies as well as unassisted conceptions. Because in vitro fertilization (IVF) cycles using eggs donated from younger to older women sidestep the constraints of age on pregnancy outcome, oocyte quality is the true driver of reproductive outcome. Experience with nuclear transfer procedures has further confirmed that the so-called biological clock rests with the egg nucleus, not the cytoplasm [1]. Since chromosomal competency of embryos is central to healthy pregnancy from IVF, it is not surprising that the highest-quality embryos (i.e., those most likely to implant) tend to be produced from eggs obtained from optimally-perfused ovaries [2,3]. But how might the efficacy of perfusion be modified to improve the therapeutic response to assisted fertility treatments? 
Platelets are small cytoplasmic cell bodies released by megakaryocytes following local injury or other physiological triggers. Traditionally considered to be first responders orchestrating hemostasis, platelets have gained renewed attention for their roles in tissue repair, neovascularization, and immunomodulation [4]. Within the platelet interior are alpha granules containing numerous growth factors, which play central roles in tissue remodeling and repair. Plateletrich plasma (PRP) has diverse clinical uses via the placement of supraphysiological concentrations of autologous growth factors at injury sites [5]. The use of PRP in the context of reproduction was first described by Pantos et al. [6]. In that report, a group of eight poor-prognosis infertility patients underwent laparoscopic intraovarian injection of PRP followed by IVF, resulting in pregnancy after thawed embryo transfer derived from non-donor oocytes. Treatment methods changed somewhat after the first registered clinical trial of intraovarian PRP, and further potential modifications and improvements continue to be investigated.

The current report aims to build on information describing PRP use in other clinical contexts and to introduce this process for reproductive endocrinology. Although a limited number of publications describing outcomes following ovarian PRP exist from small series and early case reports, no unifying theory to explain such observed reproductive effects has yet been established. Here, a novel mechanism of action is proposed based on preliminary findings after intraovarian injection of autologous activated platelet-derived growth factors.

\section{Representative technique}

\section{Baseline evaluation}

A welcome packet containing instructions and relevant medical literature should be sent to each patient prior to her appointment. Seven to 10 days before planned treatment, ovarian reserve is estimated by assessing levels of serum anti-Müllerian hormone (AMH), folliclestimulating hormone ( $\mathrm{FSH})$, and estradiol $\left(\mathrm{E}_{2}\right)$. Additionally, general metabolic status is assessed by measuring the patient's thyroid-stimulating hormone level and taking a complete blood count (including a platelet count) as well as measuring fasting prolactin, insulin, and plasminogen activator inhibitor- 1 levels. Regarding chronic pathologies, the patient must be medically cleared for intraovarian PRP injection (and possible IVF and pregnancy, where appropriate). For any patient with a body mass index of $\geq 30 \mathrm{~kg} / \mathrm{m}^{2}$ or a history of inadequate ovarian imaging, pelvic ultrasonography is recommended to ensure that both ovaries can be visualized and safely accessed via a transvaginal approach. Patients should be apprised of any likely difficulty in completing injection into one or both ovaries and written informed consent should be obtained before undergoing this investigational procedure.

\section{Sample preparation and ovarian injection}

Approximately $10 \mathrm{~mL}$ of blood is collected via peripheral venipuncture using a 21-G butterfly catheter affixed via a vacutainer to negative-pressure receiving tubes (Regen Lab, Le Mont-sur-Lausanne, Switzerland). Labeled samples are centrifuged at $1,500 \mathrm{~g} \times 5$ minutes [7]. After centrifugation, the blood is fractionated, with erythrocytes trapped below the gel and lower-density components stratified above the surface of the separator plug. Next, $<3 \mathrm{~mL}$ of supernatant (corresponding to the relatively platelet-poor plasma fraction) is aspirated off the top of each column, after which the vial is recapped for resuspension by gentle inversion. PRP activation is achieved with calcium gluconate, similar to previously-published protocols $[7,8]$. In brief, syringes are used to divide activated samples into two equal leukocyte-poor portions and are then attached to a $35-\mathrm{cm}$ single-lumen 19-G needle assembly (Rocket Medical, Washington, UK). This apparatus could be adjusted (Rickers' modification) for office PRP administration by bypassing the collection port to allow direct injection into the ovarian stroma under transvaginal ultrasound guidance. The ovaries should be aligned with the needle guide to avoid intervening vascular or other structures; the needle should then be quickly advanced without rotation deep into the central ovary. Once tip placement is confirmed, the activated PRP sample is introduced by slowpush injection as the needle is slowly withdrawn. The final $1 \mathrm{~mL}$ of the sample is deposited just under the ovarian capsule as the needle clears the ovary. After bilateral injection of activated PRP, an ultrasound assessment is performed to ensure vascular integrity and the absence of free pelvic fluid, as is done after IVF oocyte retrieval. No sedation or anesthesia is required for the ovarian injection, which can be completed in under 7 minutes. After the procedure, each patient should be asked to rest for about 15 minutes, and vital signs should be checked again before discharge.

\section{Posttreatment assessment}

To document ovarian response after intraovarian PRP injection, patients should undergo periodic testing for serum $\mathrm{AMH}, \mathrm{E}_{2}$, and $\mathrm{FSH}$ at approximately 2- to 4-week intervals. The original clinical trial enrolled menopausal or peri-menopausal patients in whom serum FSH and $E_{2}$ could be measured randomly, without respect to the day of the cycle. This testing schedule should be adjusted when patients who were acyclic at study entry begin menstruating after ovarian PRP. In those cases, we recommend testing on cycle day 2 or 3 (for $\mathrm{FSH}$ and $\mathrm{E}_{2}$ ) and retesting 2 weeks later. Limited data on serum $\mathrm{AMH}$ or FSH responsiveness following PRP dosing are currently available. However, when improvements in ovarian reserve (i.e., increased AMH or decreased FSH) are measured on two consecutive tests, patients should be contacted and encouraged to consider IVF treatment while the AMH level is still elevated relative to baseline. 


\section{Discussion}

\section{Ovarian hypoperfusion}

The connection between ovarian oxygenation and oocyte-related factors was clarified when perifollicular vascularity measured by color Doppler ultrasonography was correlated with intrafollicular dissolved oxygen content via analysis of follicular fluid obtained during egg collection for IVF. Not surprisingly, ovarian perfusion closely matches the intrafollicular oxygen level, and follicles with the lowest dissolved oxygen levels most often produce oocytes with cytoplasmic and chromosomal disorders [2,3]. Furthermore, this disruption appears to persist downstream, such that the embryos most likely to implant originate from follicles that were well-vascularized and the most highly oxygenated [2,3]. A hypoxic microenvironment thus places undesirable stress on ovarian function in general and oocyte physiology in particular.

Allied research in gerontology, nutrition, lipid/free-radical chemistry, and regenerative medicine has given new insights into how this might work. Kim et al. [9] were the first to link improved follicular perfusion with better IVF outcomes, and it seems plausible that low oxygen saturation in the follicular fluid of older and poorly perfused ovaries could amplify various local cell death pathways, promoting apoptosis and necrosis. Indeed, disordered endothelial function in polycystic ovaries is associated with microvascular dysfunction and an inhibited vasodilatory response [10], likewise leading to relatively poor outcomes. Curtailing the nutrient supply to the follicle can result in the production of reactive oxygen species (ROS), with implications for the health of both granulosa cells and oocytes.

\section{Oxidative stress}

The corrosive impact of intrafollicular ROS is difficult to overstate, extending to interference with meiotic spindle formation, which is notably attenuated and sometimes lacking entirely [11]. Experimental work with animal oocytes in vitro has shown that oxidative stress is linked to altered DNA methylation, early apoptosis, and the disruption of critical regulators of meiosis and fertilization [12-14]. ROS cutoff levels in the follicular fluid have been calculated for selected developmental benchmarks, and a threshold value appears to exist above which viable embryo formation is not favorable [15]. Even for patients not seeking pregnancy, hypoxia-mediated germ cell depletion is relevant, as it may also hasten the onset of menopause [16]. Although the full set of effects of reduced vascular flow, limited ovarian perfusion, and insufficient follicular oxygen on reproductive pathology awaits additional study, any therapy aiming to improve ovarian vascularity could represent a useful intervention. It is with this understanding that intraovarian injection of platelet growth factors was developed.
While younger eggs can utilize a range of molecular mechanisms to ameliorate oxidative damage and thus conserve germline fidelity, oocyte senescence is characterized by the blunted clearance of ROS, decreased DNA repair, reduced sensitivity of the spindle assembly checkpoint, and decreased capacity for protein repair and degradation [17]. Thus, any degree of age-related ovarian hypoperfusion further hampers the older oocyte's already-enfeebled ability to mitigate such microstructural damage.

One relatively new and highly specialized clinical setting that reveals the role of properly perfused ovarian tissue is among cancer patients who undergo surgical ovarian tissue reattachment. Graft survival hinges on reestablishing adequate vascular support, and optimization of the functional capacity of frozen-thawed ovarian tissue autografts is required for surgery to succeed. This is because cryopreserved implants are expected to sustain considerable tissue ischemia until capillary perfusion is restored after thawing, and this ischemia causes significant follicular loss not unlike that which occurs in benign ovarian aging. In 2013, successful adnexal injection of plateletderived growth factors (via PRP) was reported for posttransplant neogenesis to boost the viability of ovarian tissue autografts [18]. PRP was later shown to prevent ischemia and reperfusion damage [19]. Application of platelet-derived growth factors to help achieve necessary blood flow for grafted ovarian tissue closely aligns with PRP use prior to fertility treatment, where avoidance of low oxygen perfusion is also desired.

\section{Platelet-derived growth factors}

\section{Nuclear effects}

Growth factors released by platelets modulate tissue regeneration, cell proliferation and migration, extracellular matrix remodeling, programmed cell death, differentiation, and angiogenesis [20]. After ovulation, platelets help coordinate local tissue repair from capsular microtrauma in the adult human ovary and likely also exert wider effects on tissue remodeling [21]. Preliminary evidence supports the use of intraovarian injection of autologous PRP in managing reduced ovarian reserve $[22,23]$. Such successful cases could be explained by the intraovarian release of many soluble mediators that trigger or facilitate angiogenesis, including vascular endothelial growth factor, basic fibroblast growth factor, platelet-derived growth factors, transforming growth factor, platelet-derived angiogenesis factor, and epidermal growth factor, as well as several interleukins and insulin-like growth factor $[24,25]$.

However, if the ovarian perfusion dynamic configured by this array of platelet-derived growth factors merely amplifies the number of oocytes retrieved from aged ovaries, then nothing clinically meaningful is achieved when genetic imbalances persist in all eggs col- 


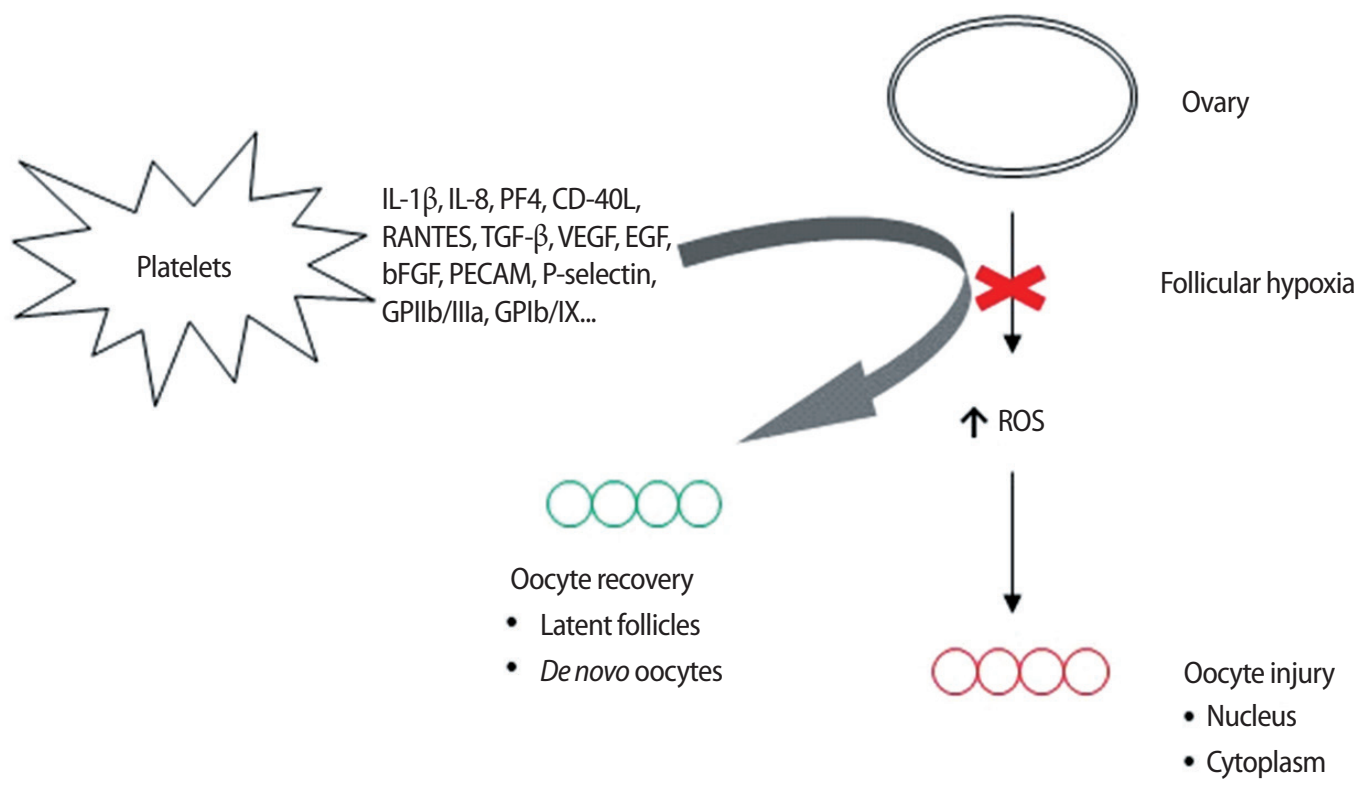

Figure 1. Proposed mechanism of action for platelet-derived growth factors inserted into ovarian tissue affected by hypoperfusion. In the absence of vascular relief secondary to signaling which facilitates improved $\mathrm{O}_{2}$ delivery, compromised follicular circulation leads to nuclear and cytoplasm pathology in oocytes. Relevant mediators in platelet releaseate include Interleukin-1 $\beta$ (IL-1 $\beta$ ), an inflammatory marker involved in cell growth, differentiation, and programmed death; IL-8 which initiates angiogenesis, perfusion, and movement to injury/infection sites; platelet factor 4 (PF4) central in directing platelet aggregation and antimicrobial activity; ligand of CD40 (CD-40L), an inflammatory signal for platelets, leukocytes, and endothelium; regulated after activation of normal T-cell expressed and secreted (RANTES) which attracts monocytes; transforming growth factor beta (TGF- $\beta$ ), a transcription activator modulating genes for differentiation, chemotaxis, and proliferation and activation of immune system cells; vascular endothelial growth factor (VEGF), a signal protein stimulating blood vessel formation; insulin-like growth factors a protein group required for cell stimulation; platelet derived growth factor, critical to blood vessel growth from adjacent capillaries, mitogenesis and proliferation of mesenchymal cells including fibroblasts, osteoblasts, tenocytes, vascular smooth muscle cells and mesenchymal stem cells; epidermal growth factor (EGF), a key messenger in cell proliferation, differentiation, and survival; basic fibroblast growth factor (bFGF), a mediator with mitogenic and cell survival activities including embryonic development, cell growth, morphogenesis, and tissue repair. Platelet expressed antigens include platelet endothelial cell adhesion molecule (PECAM), which removes aged neutrophils from circulation; P-selectin which contributes to initial recruitment of leukocytes to injury sites during inflammation; glycoprotein Ilb/llla, part of the integrin complex found on platelets aiding in platelet activation; and glycoprotein Ib and IX (GPIb/IX) which binds von Willebrand factor, allowing platelet adhesion and platelet plug formation at sites of vessel injury. ROS, reactive oxygen species.

lected. This critique was only contradicted when research was published suggesting that platelet-derived growth factors could enable what was termed "ploidy rescue" for human embryos [26]. It was hypothesized that the injection of autologous growth factors delivered angiogenic inputs to dormant ovarian tissue, and the associated uptick in perfusion not only increased the available oxygen but also delivered an array of genetic regulatory signals either to ovarian stem cells or to latent, senescent oocytes (Figure 1). Either pathway was recognized as useful to correct upstream anomalies in the oocyte-toembryo transition, including ovarian stem cell differentiation, mitochondrial dynamics, mRNA storage, translation, and degradation.

Further research is underway to elucidate how cytokines produced by and isolated from activated platelets impact chromosomal competency during oogenesis. Related experimental in vitro research in dental surgery has identified PRP-promoted cell-specific migration, proliferation, and tenogenic differentiation after upregulation of scleraxis in oral target tissue [27]. Gene expression patterns specific to tissue maturation were mimicked in PRP-treated cartilage; these patterns included downregulation of collagen types II and X and upregulation of deiodinase II and netrin-1 [28]. In addition, PRP has been implicated as a regulatory mediator of CCAAT/enhancer binding protein $\beta$ and E2F transcription factor 1 [29]. Corollary work with adult human ovarian tissue will help clarify the actions of plateletderived growth factors on oocyte genetics.

\section{Ooplasm effects}

Older ooplasm generally contains relatively few mitochondria, while older eggs engage in impaired fertilization and the embryos to which they contribute exhibit poor development, perhaps in part due to altered mitochondrial function [30]. Of note, a relatively high 
mitochondrial DNA copy number is often seen among aneuploid embryos [31], and this has been found to correspond to a relatively low chance of producing an ongoing pregnancy [32]. If enhanced oxygen perfusion after intraovarian injection of platelet-derived growth factors results in improved intrafollicular oxygen and better ooplasm quality, then the subsequent recovery of mitochondrial function could be another pathway by which ploidy rescue of blastocysts is attained.

\section{Conclusion}

The full range of factors causing ovarian insufficiency may never be known at the molecular level, but oxygen perfusion likely plays an outsized role. The fact that women who smoke experience menopause earlier than matched non-smoking controls illustrates the catastrophic results of oxygen starvation on the ovary [33]. Our approach embraces this concept with the goal of filling a crucial deficiency, aiming to diminish the need for both donor oocytes and conventional hormone replacement therapy [34].

Follicular vascularity and oxygen content in the microenvironment of the developing oocyte are increasingly recognized as critical determinants of egg competence. Insufficient oxygenation from dampened follicular perfusion results in oxidative stress and tissue injury, which translates to developmental disturbances throughout the oocyte. The angiogenic features of platelet-derived cytokines may assuage ovarian hypoxia by facilitating improved vascularity concurrent with the correction of dysregulated gene function. Initial reports have shown that intraovarian injection of PRP and/or platelet-derived growth factors (as a cell-free substrate) can bring reproductive outcomes once thought impossible, including healthy live births, within reach.

Interestingly, downregulation of genes that control DNA repair and damage response pathways may result from antecedent hypoperfusion injury before birth. The developing ovary is highly sensitive to such gestational hypoxia, with implications for the fertility of nextgeneration offspring [35-37]. Exposure to chronic hypoxia during fetal development accelerates ovarian aging and decreases reserve in adulthood. While synthetic gonadotropins used for follicular recruitment are an important component of advanced reproductive technologies, these medications represent pharmacological brushstrokes on a blank ovarian canvas. Platelet-derived growth factors, when applied appropriately, seem to prepare the field to optimize controlled ovulation induction. Additional investigation focusing on the ovary (including ovarian stem cells) is needed to define which platelet-derived signals are most involved in oocyte replenishment and ploidy rescue, thereby offering new methods to make IVF successful for those patients with the lowest ovarian reserve. Further studies of the ovarian germline stem cell niche and its requisite regulatory inputs promise to yield valuable insights into reproductive aging.

\section{Conflict of interest}

ESS has issued a provisional U.S. patent for a technique involving intraovarian autologous platelet-rich plasma use. No other potential conflicts of interest relevant to this article were reported.

\section{ORCID}

\section{E. Scott Sills https://orcid.org/0000-0001-7334-1031}

\section{Author contributions}

Conceptualization, Data curation, Formal analysis, Project administration, Visualization, Writing-original draft, review \& editing: all authors.

\section{References}

1. Keefe DL. Telomeres, reproductive aging, and genomic instability during early development. Reprod Sci 2016;23:1612-5.

2. Van Blerkom J. Epigenetic influences on oocyte developmental competence: perifollicular vascularity and intrafollicular oxygen. J Assist Reprod Genet 1998;15:226-34.

3. Borini A, Maccolini A, Tallarini A, Bonu MA, Sciajno R, Flamigni C. Perifollicular vascularity and its relationship with oocyte maturity and IVF outcome. Ann NY Acad Sci 2001;943:64-7.

4. Fernandez-Moure JS, Van Eps JL, Cabrera FJ, Barbosa Z, Medrano Del Rosal G, Weiner BK, et al. Platelet-rich plasma: a biomimetic approach to enhancement of surgical wound healing. J Surg Res 2017;207:33-44.

5. Dhillon RS, Schwarz EM, Maloney MD. Platelet-rich plasma therapy: future or trend? Arthritis Res Ther 2012;14:219.

6. Pantos K, Nitsos N, Kokkali G, Vaxevanoglou T, Markomichali C, Pantou $A$, et al. Ovarian rejuvenation and folliculogenesis reactivation in peri-menopausal women after autologous plateletrich plasma treatment. ESHRE 32nd annual meeting; $2016 \mathrm{Jul}$ 3-6; Helsinki, Finland. Hum Reprod 2016;Suppl 1:i301.

7. Gkini MA, Kouskoukis AE, Tripsianis G, Rigopoulos D, Kouskoukis $K$. Study of platelet-rich plasma injections in the treatment of androgenetic alopecia through an one-year period. J Cutan Aesthet Surg 2014;7:213-9.

8. Vahabi S, Yadegari Z, Mohammad-Rahimi H. Comparison of the effect of activated or non-activated PRP in various concentrations on osteoblast and fibroblast cell line proliferation. Cell Tis- 
sue Bank 2017; 18:347-53.

9. Kim KH, Oh DS, Jeong JH, Shin BS, Joo BS, Lee KS. Follicular blood flow is a better predictor of the outcome of in vitro fertilization-embryo transfer than follicular fluid vascular endothelial growth factor and nitric oxide concentrations. Fertil Steril 2004; 82:586-92.

10. Lakhani K, Leonard A, Seifalian AM, Hardiman P. Microvascular dysfunction in women with polycystic ovary syndrome. Hum Reprod 2005;20:3219-24.

11. Chattopadhayay R, Ganesh A, Samanta J, Jana SK, Chakravarty $\mathrm{BN}$, Chaudhury K. Effect of follicular fluid oxidative stress on meiotic spindle formation in infertile women with polycystic ovarian syndrome. Gynecol Obstet Invest 2010;69:197-202.

12. Tao Y, Liu XJ. Deficiency of ovarian ornithine decarboxylase contributes to aging-related egg aneuploidy in mice. Aging Cell 2013;12:42-9.

13. Menezo YJ, Silvestris E, Dale B, Elder K. Oxidative stress and alterations in DNA methylation: two sides of the same coin in reproduction. Reprod Biomed Online 2016;33:668-83.

14. Zhang M, Miao Y, Chen Q, Cai M, Dong W, Dai X, et al. BaP exposure causes oocyte meiotic arrest and fertilization failure to weaken female fertility. FASEB J 2018;32:342-52.

15. Jana SK, K NB, Chattopadhyay R, Chakravarty B, Chaudhury K. Upper control limit of reactive oxygen species in follicular fluid beyond which viable embryo formation is not favorable. Reprod Toxicol 2010;29:447-51.

16. Yadav AK, Yadav PK, Chaudhary GR, Tiwari M, Gupta A, Sharma A, et al. Autophagy in hypoxic ovary. Cell Mol Life Sci 2019;76:331122.

17. Mihalas BP, Redgrove KA, McLaughlin EA, Nixon B. Molecular mechanisms responsible for increased vulnerability of the ageing oocyte to oxidative damage. Oxid Med Cell Longev 2017; 2017:4015874.

18. Callejo J, Salvador C, Gonzalez-Nunez S, Almeida L, Rodriguez L, Marques $L$, et al. Live birth in a woman without ovaries after autograft of frozen-thawed ovarian tissue combined with growth factors. J Ovarian Res 2013;6:33.

19. Bakacak M, Bostanci MS, Inanc F, Yaylali A, Serin S, Attar R, et al. Protective effect of platelet rich plasma on experimental ischemia/reperfusion injury in rat ovary. Gynecol Obstet Invest 2016; 81:225-31.

20. Gurtner GC, Werner S, Barrandon Y, Longaker MT. Wound repair and regeneration. Nature 2008;453:314-21.

21. Lacci KM, Dardik A. Platelet-rich plasma: support for its use in wound healing. Yale J Biol Med 2010;83:1-9.

22. Sfakianoudis K, Simopoulou M, Nitsos N, Rapani A, Pantou A, Vaxevanoglou T, et al. A case series on platelet-rich plasma revo- lutionary management of poor responder patients. Gynecol Obstet Invest 2019;84:99-106.

23. Sills ES, Rickers NS, Li X, Palermo GD. First data on in vitro fertilization and blastocyst formation after intraovarian injection of calcium gluconate-activated autologous platelet rich plasma. Gynecol Endocrinol 2018;34:756-60.

24. Lubkowska A, Dolegowska B, Banfi G. Growth factor content in PRP and their applicability in medicine. J Biol Regul Homeost Agents 2012;26(2 Suppl 1):3S-22S.

25. Martínez CE, Smith PC, Palma Alvarado VA. The influence of platelet-derived products on angiogenesis and tissue repair: a concise update. Front Physiol 2015;6:290.

26. Sills ES, Rickers NS, Svid CS, Rickers JM, Wood SH. Normalized ploidy following 20 consecutive blastocysts with chromosomal error: healthy 46, XY pregnancy with IVF after intraovarian injection of autologous enriched platelet-derived growth factors. Int J Mol Cell Med 2019;8:84-90.

27. Imai S, Kumagai K, Yamaguchi Y, Miyatake K, Saito T. Platelet-rich plasma promotes migration, proliferation, and the gene expression of scleraxis and vascular endothelial growth factor in paratenon-derived cells in vitro. Sports Health 2019;11:142-8.

28. Zhang Y, Morgan BJ, Smith R, Fellows CR, Thornton C, Snow M, et al. Platelet-rich plasma induces post-natal maturation of immature articular cartilage and correlates with LOXL1 activation. Sci Rep 2017;7:3699.

29. Shen $H$, Cheng $H$, Chen $H$, Zhang J. Identification of key genes induced by platelet-rich plasma in human dermal papilla cells using bioinformatics methods. Mol Med Rep 2017;15:81-8.

30. Sills ES, Takeuchi T, Tucker MJ, Palermo GD. Genetic and epigenetic modifications associated with human ooplasm donation and mitochondrial heteroplasmy: considerations for interpreting studies of heritability and reproductive outcome. Med Hypotheses 2004;62:612-7.

31. Jing Y, Li L, LiYY, Ouyang YC, Sun QY, Zhang CL, et al. Embryo quality, and not chromosome nondiploidy, affects mitochondrial DNA content in mouse blastocysts. J Cell Physiol 2019;234:10481-8.

32. Lledo B, Ortiz JA, Morales R, Garcia-Hernandez E, Ten J, Bernabeu $A$, et al. Comprehensive mitochondrial DNA analysis and IVF outcome. Hum Reprod Open 2018;2018:hoy023.

33. Whitcomb BW, Purdue-Smithe AC, Szegda KL, Boutot ME, Hankinson SE, Manson JE, et al. Cigarette smoking and risk of early natural menopause. Am J Epidemiol 2018;187:696-704.

34. Sills ES, Li X, Rickers NS, Wood SH, Palermo GD. Metabolic and neurobehavioral response following intraovarian administration of autologous activated platelet rich plasma: first qualitative data. Neuro Endocrinol Lett 2019;39:427-33.

35. Oliveira PH, Boura JS, Abecasis MM, Gimble JM, da Silva CL, Ca- 
bral JM. Impact of hypoxia and long-term cultivation on the genomic stability and mitochondrial performance of ex vivo expanded human stem/stromal cells. Stem Cell Res 2012;9:225-36. 36. Aiken CE, Tarry-Adkins JL, Spiroski AM, Nuzzo AM, Ashmore TJ, Rolfo A, et al. Chronic gestational hypoxia accelerates ovarian aging and lowers ovarian reserve in next-generation adult rats. FASEB J 2019;33:7758-66.

37. Pampanini V, Jahnukainen K, Sahlin L, Germani D, Puglianiello A, Cianfarani $S$, et al. Impact of uteroplacental insufficiency on ovarian follicular pool in the rat. Reprod Biol Endocrinol 2019;17:10. 